\title{
Comparison of photodynamic efficiency of cholesterol, selected cholesterol esters, metabolites and oxidation products in lipid peroxidation processes*
}

\author{
Monika Burakowska1,2, Tadeusz Sarna1 and Anna M. Pawlak ${ }^{1 凶}$ \\ 1Department of Biophysics, Faculty of Biochemistry, Biophysics and Biotechnology, Jagiellonian University, Kraków, Poland; 2Faculty of Electrical \\ Engineering, Automatics, Computer Science and Biomedical Engineering, AGH-University of Science and Technology, Kraków, Poland
}

\begin{abstract}
Cholesterol $(\mathrm{Ch})$ is one of the most important components of biological membranes which has a significant impact on their biophysical properties. As a key component of lipid membranes, along with other unsaturated lipids present in a biological membrane $\mathrm{Ch}$ undergoes an oxidation reaction during oxidative stress. Cholesterol oxidation products, cholesteryl esters and metabolites are also localized in the lipid membranes, where they may modify the membrane properties. In this work, the impact on lipid peroxidation (induced by a photodynamic action) of cholesterol, selected cholesteryl esters, cholesterol oxidation products and metabolites has been studied using EPR oximetry and direct detection of singlet oxygen phosphorescence at $1270 \mathrm{~nm}$. The obtained rate constant values of interaction of selected lipids and sterols with singlet oxygen indicate that the tested compounds are not efficient singlet oxygen quenchers. Nevertheless, to different extents, presence of sterols modifies the oxygen photoconsumption rate in peroxidisable liposomes.
\end{abstract}

Keywords: cholesterol, cholesterol derivatives, oxidation, liposomes, singlet oxygen

Received: 20 October, 2021; revised: 30 October, 2021; accepted: 30 October, 2021; available on-line: 15 November, 2021

\section{$\bigotimes_{\text {e-mail: anna.pawlak@uj.edu.pl }}$}

*This paper has been published on the occasion of Jubilee Conference entitled "The latest achievements in biochemistry, biophysics and biotechnology - 50 years of history of the Faculty of Biochemistry, Biophysics and Biotechnology of the Jagiellonian University in Kraków" Kraków, September 23-24, 2021.

Acknowledgements of Financial Support: AP acknowledges the National Science Centre (NCN, Grant nr 2012/05/E/NZ3/00473); Faculty of Biochemistry, Biophysics and Biotechnology of Jagiellonian University, a partner of the Leading National Research Center (KNOW) supported by the Ministry of Science and Higher Education.

Abbreviations: Ch, cholesterol; $\mathrm{ChE}$, cholesterol epoxide; ChO, cholesteryl oleate; ChP, cholesteryl palmitate; EPR, electron paramagnetic resonance; $\mathrm{HSCh}$, cholesteryl hemisuccinate; 7KCh, 7-ketocholesterol; PBS, phosphate buffer saline; RB, Rose Bengal.

\section{INTRODUCTION}

Cholesterol $(\mathrm{Ch})$ is one of the most important components of biological membranes and its presence and concentration have a significant impact on their biophysical properties, such as fluidity, permeability or hydrophobicity (Subczynski et al., 2017) (Fig. 1). As a key component of lipid membranes, Ch may be exposed to a stream of free radicals or singlet oxygen during oxidative stress and along with other unsaturated lipids present in a membrane serves as a substrate for oxidation reaction (Geiger et al., 1997; Vila et al., 2001; Kulig et al., 2016). Cholesterol oxidation products, such as 7-ketocholesterol (7KCh) or 5,6-epoxy cholesterol, are also hydrophobic, and therefore they remain in the cell membranes where they may interact with other membrane components and modify the membrane properties (Kulig et al., 2016; Wnętrzak et al., 2017). 7KCh is one of the most extensively studied oxysterols and is considered to be the most cytotoxic (Anderson et al., 2020). Increased levels of $7 \mathrm{KCh}$ have been noted in the human atherosclerotic plaque (Rao et al., 2014) and in ocular tissues where it accumulates with age and after extensive exposure to light (Rodriguez \& Fliesler, 2009; Rodriguez et al., 2014). Atherosclerosis development is accompanied by accumulation of cytoplasmic lipid droplets mainly composed of cholesteryl oleate (ChO) (Brown \& Goldstein, 1983) (Fig. 1). The source of cholesteryl oleate for lipid droplets are foam cells derived from macrophages that took up oxidized low-density lipoprotein (LDL) (Enomoto et al., 1987; Nagano et al., 1991). LDL consists of a hydrophobic core of neutral lipids, mainly cholesteryl esters, surrounded by a polar surface shell and serves as the major cholesterol transporter in human plasma (Koivuniemi et al., 2009).

Cholestanol is a saturated sterol existing at a very low physiological concentration in animals (Dotti et al., 2001) (Fig. 1). However, an increase in its level

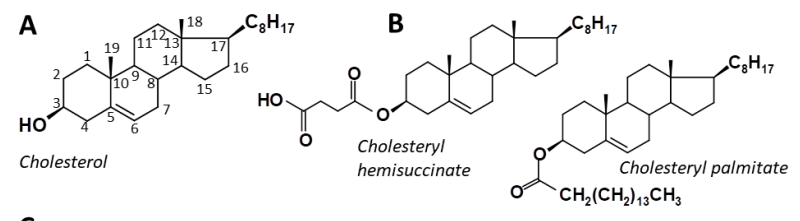

C

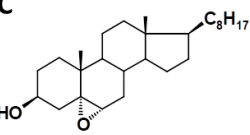

Cholesteral $5 \alpha, 6 \alpha$-epoxide
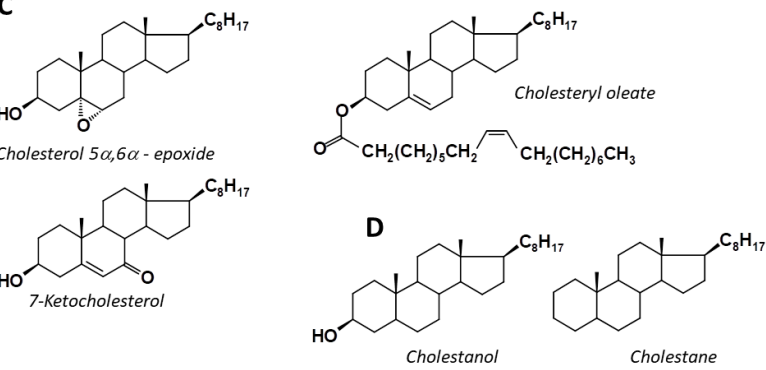

Figure 1. Chemical structures and atom numbering of the tetracyclic part of cholesterol $(A)$ and chemical structures of selected cholesteryl esters (B), oxidation products (C) and metabolites (D). 
occurs along with the development of a hereditary disease, the cerebrotendinous xanthomatosis (CTX) (Dotti et al., 2001; Seyama, 2003). Cholestanol deposition causes neurological dysfunction, mental retardation and juvenile cataract (Bhattacharyya et al., 2007). It has been proposed that the mechanism responsible for cell damage in CTX involves an imbalance of the cholesterol/cholestanol ratio in the plasma membrane and structural changes caused by cholestanol, which in turn disturb the calcium channel function of the membrane (Seyama, 2003).

It seems that physiologically relevant sterols localized in the lipid membranes exert a significant impact on their biophysical properties and function. In this work, the effect of cholesterol, selected cholesteryl esters, cholesterol oxidation products and metabolites on lipid peroxidation (induced by a photodynamic action) has been studied.

\section{MATERIAL AND METHODS}

Cholesterol, 1,2-dimyristoyl-sn-glycero-3-phosphocholine ((14:0)(14:0)PC), DMPC; 1-palmitoyl-2-oleoyl-glycero-3-phosphocholine ((16:0)(18:1)PC), POPC; and 1-palmitoyl-2-docosahexaenoyl-sn-glycero-3-phosphatidylcholine ((16:0)(22:6)PC) were purchased from Avanti Polar Lipids, Inc. (Alabaster Alabama, USA).

Cholesteryl oleate (18:1 Chol Ester); cholesteryl palmitate (16:0 Chol Ester); Cholesteryl hemisuccinate (HSCh); $5 \alpha$-cholestan-3 $\beta$-ol (cholestanol); 5- $\alpha$-Cholestane (Cholestane); 7-ketocholesterol (7KCh), cholesterol $5 \alpha, 6 \alpha$-epoxide, Rose bengal (RB), and 5,10, 15,20-tetraphenyl-21H,23H-porphine (TPP) were purchased from Sigma-Aldrich Inc.

Sodium phosphate, potassium phosphate, sodium chloride, potassium chloride, liquid chromatography grade benzene, chloroform, and methanol were purchased from Avantor Performance Materials Poland (Gliwice, Poland) and used as supplied.

4-Protio-3-carbamoyl-2,2,5,5-tetraperdeuteromethyl3-pyrroline-1-yloxy (mHCTPO) was a gift from Howard J. Halpern (University of Chicago, Chicago, IL).

\section{Preparation of multilamellar liposomes}

Multilamellar liposomes were prepared by the film deposition method as previously described (Nayar, 1989; MacDonald et al., 1991; Subczynski et al., 2007). Briefly: selected synthetic lipids were dissolved in chloroform which was then evaporated with a stream of neutral gas (nitrogen or argon). The lipid film formed on the bottom of the glass tube was completely dried under reduced pressure for 2-4 h. A phosphate buffer saline (PBS, $10 \mathrm{mM}, \mathrm{pH}$ 7.4), previously incubated for at least $24 \mathrm{~h}$ with chelex to remove a trace of transition metal ions, was added to the dried lipid film at room temperature, in small portions to the final volume. During preparation, the samples were vortexed vigorously to completely remove the lipid film from the test tubes.

In these studies, liposomes with two slightly different lipid compositions were prepared. One type of liposomes comprised of POPC (16 mM), DMPC (2 mM) and $\mathrm{Ch}(2 \mathrm{mM})$ or its selected esters, oxidation products or metabolites. The second type of liposomes tested in this work was composed of partly oxidised (16:0)(22:6) PC (final concentration $9 \mathrm{mM})$, DMPC $(2 \mathrm{mM})$ and $\mathrm{Ch}$ or its selected esters, oxidation products or metabolites (1 $\mathrm{mM})$.
The final concentrations of synthetic lipids in both suspensions of multilamellar liposomes (MLV) were 20 $\mathrm{mM}$ and $12 \mathrm{mM}$, respectively. All preparations were performed in darkness or under dim light and, where possible, under nitrogen.

\section{Singlet Oxygen Quenching Measurements}

To determine the rate constants of the interactions of singlet oxygen with cholesterol and its selected esters, oxidation products or metabolites, time-resolved direct detection of singlet oxygen $\left({ }^{1} \mathrm{O}_{2},{ }^{1} \Delta_{\mathrm{g}}\right)$ phosphorescence at $1270 \mathrm{~nm}$ was used. In these measurements, changes in singlet oxygen lifetime were monitored as a function of the sterol concentration. Tetraphenylporphyrine (TPP) was used as an efficient singlet oxygen generator, with quantum yield up to 0.6 in benzene (Bonnett et al., 1988; Redmond \& Gamlin, 1999). Absorbance of TPP in a chloroform solution was adjusted to 0.05 at the excitation wavelength $(\lambda=645 \mathrm{~nm})$. Sensitizer solution was placed in a quartz fluorescence cuvette (QA-1000; Hellma, Mullheim, Germany) and excited with $645 \mathrm{~nm}$ light generated by an integrated nanosecond DSS Nd:YAG laser system (NT242-1k-SH/SFG; Ekspla, Vilnius, Lithuania). The near-infrared singlet oxygen phosphorescence was measured perpendicularly to the excitation beam in a photon-counting mode using a thermoelectric cooled NIR PMT module (H10330-45; Hamamatsu Photonics, Hamamatsu, Japan). Measurements were repeated with increasing concentrations of cholesterol, its selected esters, oxidation products or metabolites. Data analysis, including first-order ${ }^{1} \mathrm{O}_{2}\left({ }^{1} \Delta_{\mathrm{g}}\right)$ phosphorescence decay fitted by the Levenberg-Marquardt algorithm, was performed by custom-written software.

\section{EPR Oximetry}

To determine photo-induced oxygen uptake in peroxidizable liposomes in the presence of cholesterol or its selected esters, oxidation products or metabolites (10 or $8.3 \mathrm{~mol} \%$ ), electron paramagnetic resonance oximetry was employed. As the oxygen-sensitive spin probe, mHCTPO $(0.1 \mathrm{mM})$ was utilized. Samples (final volume of $200 \mu \mathrm{L}$ ) for oxygen consumption rate measurements consisted of previously prepared liposomes $(150 \mu \mathrm{L})$, solution of exogenous sensitizer Rose Bengal (RB) $(10 \mu \mathrm{M}, 10 \mu \mathrm{L})$ and a spin probe $(20 \mu \mathrm{L}$, final concentration $0.1 \mathrm{mM})$ in PBS $(10 \mathrm{mM}, \mathrm{pH} 7.4,20 \mu \mathrm{L})$. In case of liposomes containing partly oxidised (16:0)(22:6) PC, no sensitizer was used, and a volume of RB solution was replaced by $10 \mu \mathrm{L}$ of PBS. Measurements were carried out in a flat quartz cell placed in the EPR resonant cavity as previously described (Rozanowska et al., 1995; Zadlo et al., 2009), employing the following instrument settings: microwave power $1.06 \mathrm{~mW}$, modulation amplitude $0.006 \mathrm{mT}$, scan width $0.3 \mathrm{mT}$, and scan time $5.2 \mathrm{~s}$, using a Bruker EMX-AA 1579 EPR spectrometer (Bruker BioSpin, Rheinstetten, Germany). Liposomes containing $\mathrm{RB}$ or partly oxidised (16:0)(22:6)PC were irradiated in situ during measurements with green (516-586 nm; $\left.44 \mathrm{~mW} / \mathrm{cm}^{2}\right)$ or blue $\left(404-515 \mathrm{~nm} ; 55 \mathrm{~mW} / \mathrm{cm}^{2}\right)$ light, respectively.

\section{Statistical Analysis}

Statistical analysis was performed by Student's $t$ test, and linear regression was performed by the method of least squares. 
Table 1. Oxygen uptake rates ( $\mathrm{mM} / \mathrm{min})$ in liposomes containing POPC (16 $\mathrm{mM})$, DMPC (2mM) and cholesterol or selected cholesteryl eters, cholesterol oxidation products or cholesterol metabolites ( $2 \mathrm{mM})$.

Oxygen photo consumption induced in liposomes irradiated with green light (516-586 nm; $\left.44 \mathrm{~mW} / \mathrm{cm}^{2}\right)$ in the presence of sensitizer Rose Bengal $(10 \mu \mathrm{M})$.

Sample

Incubated in the dark

Photoinduced oxygen uptake rate $(\mathrm{mM} / \mathrm{min})$ in the presence of $\mathrm{Rb}(10 \mu \mathrm{M})$

\begin{tabular}{|c|c|c|c|}
\hline & & liated with green I & \\
\hline \multicolumn{2}{|l|}{ Cholesterol } & $(6.73 \pm 1.02) \times 10^{-5}$ & $0.320 \pm 0.100$ \\
\hline \multirow{3}{*}{ Cholesteryl esters } & cholesteryl palmitate & $(3.38 \pm 1.23) \times 10^{-5}$ & $0.152 \pm 0.043$ \\
\hline & cholesteryl oleate & $(3.57 \pm 0.45) \times 10^{-4}$ & $0.148 \pm 0.054$ \\
\hline & cholesteryl hemisuccinate & $(1.57 \pm 0.35) \times 10^{-4}$ & $0.176 \pm 0.035$ \\
\hline \multirow{2}{*}{ Cholesterol oxidation products } & 7-ketocholesterol & $(2.32 \pm 0.87) \times 10^{-4}$ & $0.353 \pm 0.038$ \\
\hline & cholesterol epoxide & $(5.18 \pm 1.05) \times 10^{-5}$ & $0.329 \pm 0.041$ \\
\hline \multirow{2}{*}{ Cholesterol metabolities } & cholestanol & $(2.15 \pm 0.86) \times 10^{-4}$ & $0.401 \pm 0.094$ \\
\hline & cholestane & $(1.11 \pm 0.50) \times 10^{-4}$ & $0.202 \pm 0.054$ \\
\hline
\end{tabular}

\section{RESULTS AND DISCUSSION}

\section{Green-light induced oxygen consumption in the presence of Rose Bengal}

The EPR oximetry measurements enabled to compare the impact of cholesterol, selected cholesteryl esters, cholesterol oxidation products and cholesterol metabolites on the rate of oxygen consumption $\left(\mathrm{d}\left[\mathrm{O}_{2}\right] / \mathrm{dt}\right)$ in liposome samples of two types in respect to their composition and peroxidisability, undergoing oxidation induced by two different agents. In the first type of liposomes,

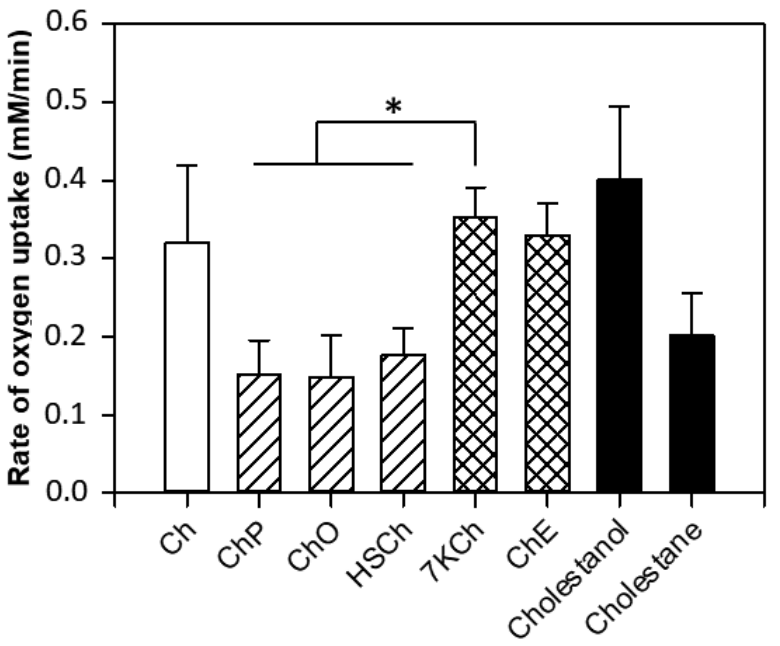

Figure 2. Oxygen consumption rates in liposomal samples composed of DMPC ( $2 \mathrm{mM})$, POPC (16 mM) and $2 \mathrm{mM}$ cholesterol (empty bar) or one of its esters (diagonal stripes), oxidation products (diagonal check) or metabolites (black bars).

All bars represent green light-induced (516-586 nm; $44 \mathrm{~mW} / \mathrm{cm}^{2}$ ) initial rates of oxygen uptake $(\mathrm{mM} / \mathrm{min})$ in the presence of Rose Bengal $(10 \mu \mathrm{M})$. Oxygen consumption rate in the dark-incubated samples did not exceed $4 \times 10^{-4} \mathrm{mM} / \mathrm{min}$ (not shown). Differences in the oxygen photo-uptake rate between 7KCh and each of cholesteryl esters are statistically significant $(P<0.05)$ comprised of POPC, DMPC and cholesterol or its derivative or analogue, oxygen consumption was induced by irradiation with green light (516-586 nm; $44 \mathrm{~mW} /$ $\left.\mathrm{cm}^{2}\right)$ in the presence of Rose Bengal $(10 \mu \mathrm{M})$. Results of oxygen photo-uptake measurements in liposomes, subjected to RB-photosensitized oxidation are presented in Fig. 2 and Table 1.

The rate of oxygen consumption $\left(\mathrm{d}\left[\mathrm{O}_{2}\right] / \mathrm{dt}\right)$ measured in the dark was very low and did not exceed $4 \times 10^{-4}$ $(\mathrm{mM} / \mathrm{min})$, indicating that oxygen was hardly consumed, and lipids did not undergo significant oxidation. Similar values were obtained for green light irradiated samples without a photosensitizer (not shown). The oxygen uptake rates measured in irradiated samples containing a sensitizer were the highest in liposomes comprised of cholestanol $(0.401 \pm 0.094 \mathrm{mM} / \mathrm{min})$, 7-ketocholesterol $(0.353 \pm 0.038 \mathrm{mM} / \mathrm{min})$, cholesterol $5 \alpha, 6 \alpha$ - epoxide $(0.329 \pm 0.041)$ and cholesterol $(0.320 \pm 0.100 \mathrm{mM} / \mathrm{min})$. The presence of cholestane reduced oxygen uptake rate to $0.202 \pm 0.054 \mathrm{mM} / \mathrm{min}$, while the lowest values of $\mathrm{d}\left[\mathrm{O}_{2}\right] / \mathrm{dt}$ were observed in the presence of cholesteryl esters: $0.176 \pm 0.035,0.152 \pm 0.043$ and $0.148 \pm 0.054 \mathrm{mM} /$ min for cholesteryl hemisuccinate, cholesteryl palmitate and cholesteryl oleate, respectively (Fig. 2, Table 1). $\mathrm{RB}$ is an efficient, water soluble photosensitizer that can generate both, the singlet oxygen and free radicals upon irradiation with visible/green light (Neckers, 1989; Różanowska et al., 1995; DeRosa \& Crutchley, 2002). It has been shown that quantum yield of singlet oxygen generation by $\mathrm{RB}$ in PBS solutions reaches up to 0.75 (Redmond \& Gamlin, 1999). Although RB, as a moderately hydrophobic compound (Crandon et al., 2020), easily associates with the lipid membrane (Lambert \& Kochevar, 1997), it cannot incorporate into it because it exists as a dianion in polar solvents (Stockett et al., 2020). Thus, RB generates reactive oxygen species (ROS) in the aqueous phase at the surface of the membrane, and not in the membrane itself. On the other hand, the substrates for the reaction with ROS generated by RB, peroxidizable lipids, are localised inside the lipid membrane of liposomes suspended in this aqueous solution. The lifetime of a singlet oxygen $\left({ }^{1} \mathrm{O}_{2}\left({ }^{1} \Delta_{\mathrm{g}}\right)\right)$ in water is 


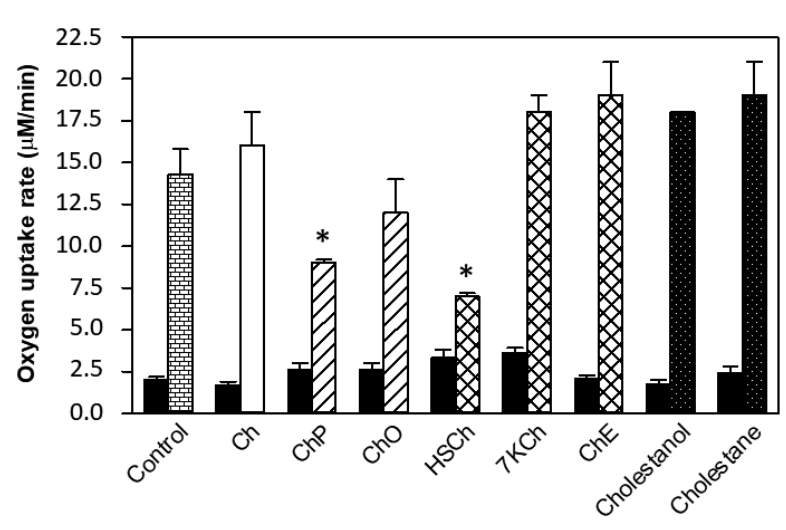

Figure 3. Oxygen consumption $(\mu \mathrm{M} / \mathrm{min}$ ) in liposomal control sample composed of DMPC (2 mM) and (16:0)(22:6)PC (9 mM) (grey bar) and in samples also containing $1 \mathrm{mM}$ cholesterol (empty bar) or one of its esters (diagonal stripes), oxidation products (diagonal check) or metabolites (black bar with white dots) irradiated with blue-light $\left(404-515 \mathrm{~nm} ; 55 \mathrm{~mW} / \mathrm{cm}^{2}\right)$.

All black bars represent oxygen uptake rate measured in samples incubated in the dark. Differences in oxygen photo-uptake rate between $\mathrm{ChP}$ - or HSCh-containing samples and the other samples are statistically significant $(P<0.05)$.

only $4 \mu$ s (Rodgers \& Snowden, 1982), and the sites of the ${ }^{1} \mathrm{O}_{2}\left({ }^{1} \Delta_{\mathrm{g}}\right)$ attack, the double bonds in the lipid molecules forming the liposome membrane, are located inside the membrane. Location and depth of immersion of cholesterol and its derivatives/analogues in the membrane depends on lipid composition of the membrane, the length of fatty acid chains esterified in phospholipids, and the degree of their unsaturation (Subczynski et al., 2012; Yang et al., 2016). The highest values of $\mathrm{d}\left[\mathrm{O}_{2}\right] / \mathrm{dt}$ were observed in liposomes containing cholesterol, cholestanol, ketocholesterol and cholesterol epoxide (Fig. 2, Table 1). All of these molecules contain a hydroxyl group, which due to its location close to the membrane surface, may increase the probability of ROS to reach inside the lipid bilayer. In the case of cholesterol esters, in which the hydroxyl group is "blocked" by a fatty acid or succinate esterified at this site, and in the case of cholestane, which is devoid of the hydroxyl group, the rates of oxygen consumption were significantly lower. There was almost a two-fold decrease in the rate of oxygen consumption in comparison to the cholesterol-containing membranes. These observations are confirmed by the results obtained in liposomes of the second type containing partially peroxidised (16:0)(22:6) PC, DMPC and Ch or its derivative/analogue and irradiated with blue light $\left(404-515 \mathrm{~nm} ; 55 \mathrm{~mW} / \mathrm{cm}^{2}\right)$.

Blue-light induced oxygen consumption in the presence of highly peroxidisable (16:0)(22:6)PC

In these samples, the lowest oxygen consumption rates were also observed in the presence of cholesterol esters. The values of $\mathrm{d}\left[\mathrm{O}_{2}\right] / \mathrm{dt}$ for $\mathrm{HSCh}, \mathrm{PCh}$ and $\mathrm{OCh}$ were as follows: $(6.9 \pm 0.2) \mu \mathrm{M} / \mathrm{min},(8,5 \pm 0.2) \mu \mathrm{M} / \mathrm{min}$ and $(11.8 \pm 2.0) \mu \mathrm{M} / \mathrm{min}$ (Fig. 3, Table 2). The highest rates of oxygen photoconsumption were observed in the presence cholestanol, cholestane, cholesterol epoxide and ketocholesterol: $(19.3 \pm 2.0) \mu \mathrm{M} / \mathrm{min},(18.3 \pm 0.04)$ $\mu \mathrm{M} / \mathrm{min}$, $(18.8 \pm 2.0) \mu \mathrm{M} / \mathrm{min}$ and $(17.7 \pm 1.0) \mu \mathrm{M} / \mathrm{min}$, respectively. Only a slightly lower value of $\mathrm{d}[\mathrm{O} 2] / \mathrm{dt}$ was recorded in the irradiated control sample, i.e. liposomes comprised of (16:0)(22:6)PC and DMPC only: (14.3 \pm 1.5$) \mu \mathrm{M} / \mathrm{min}$ (Fig. 3, Table 2). Low, but noticeable level of oxygen uptake was observed in all samples incubated in the dark, possibly due to autooxidation of highly peroxidizable (16:0)(22:6)PC. Polyunsaturated docosahexaenoic acid (DHA) esterified at the SN-2 position of this lipid plays a very important role in the protection of photoreceptor membranes in the retina of the eye (Jeffrey et al., 2001). This acid is highly susceptible to free radical peroxidation, the products of which may affect degeneration of the retina (Liu et al., 2014). Recently, it has been shown that oxidation of $(16: 0)(22: 6) \mathrm{PC}$ extends its absorption spectrum to the visible region of the electromagnetic spectrum and makes it photoreactive (Różanowska et al., 2021). Oxidized (16:0)(22:6)PC, or rather a mixture of its oxidation products, shows significant photoreactivity when exposed to short wavelength visible light. Oxidized (16:0)(22:6)PC efficiently generates singlet oxygen and free radicals under blue light illumina-

Table 2. Oxygen uptake rate $(\mu \mathrm{M} / \mathrm{min})$ in highly peroxidisable liposomes containing (16:0)(22:6)PC (9 mM), DMPC (2 mM) and cholesterol or selected cholesteryl esters, cholesterol oxidation products or cholesterol metabolites $(1 \mathrm{mM})$.

Oxygen photo consumption induced in liposomes irradiated with blue light $\left(404-515 \mathrm{~nm} ; 55 \mathrm{~mW} / \mathrm{cm}^{2}\right)$ or incubated in the dark.

Sample

Photoinduced oxygen uptake rate $(\mu \mathrm{M} / \mathrm{min})$

Incubated in the dark

Irradiated with blue light

\begin{tabular}{|c|c|c|c|}
\hline Control & & $1.99 \pm 0.2$ & $14.3 \pm 1.5$ \\
\hline Cholesterol & & $1.71 \pm 0.2$ & $15.9 \pm 2.0$ \\
\hline \multirow{3}{*}{ Cholesteryl esters } & cholesteryl palmitate & $2.62 \pm 0.4$ & $8.5 \pm 0.2$ \\
\hline & cholesteryl oleate & $2.60 \pm 0.4$ & $11.8 \pm 2.0$ \\
\hline & cholesteryl hemisuccinate & $3.32 \pm 0.5$ & $6.9 \pm 0.2$ \\
\hline \multirow{2}{*}{ Cholesterol oxidation products } & 7-ketocholesterol & $3.63 \pm 0.3$ & $17.7 \pm 1.0$ \\
\hline & cholesterol epoxide & $2.09 \pm 0.2$ & $18.8 \pm 2.0$ \\
\hline \multirow{2}{*}{ Cholesterol metabolities } & cholestanol & $1.73 \pm 0.3$ & $18.3 \pm 0.04$ \\
\hline & cholestane & $2.43 \pm 0.4$ & $19.3 \pm 2.0$ \\
\hline
\end{tabular}


tion (Różanowska et al., 2021). It is not known which oxidation products of (16:0)(22:6)PC are responsible for the observed photoreactivity and if they are present in the retina, but similar results were obtained in case of ex vivo oxidised lipids extracted from bovine retinas (Koscielniak et al., 2017; Pawlak et al., 2019).

In liposomes containing partially peroxidized (16:0) (22:6)PC, the reactive oxygen species were likely generated inside the membrane. And the main substrate for ROS generated by partially oxidised (16:0)(22:6)PC in the tested samples was mainly the phospholipid itself. In the control sample, which contained (16:0)(22:6)PC and DMPC, the only substrate for oxidation was the unsaturated lipid. Unfortunately, it was not possible to monitor the rate of oxidation/uptake of (16:0)(22:6)PC during the irradiation of the tested samples. In the presence of cholesteryl esters, a significant decrease in $\mathrm{d}\left[\mathrm{O}_{2}\right] / \mathrm{dt}$ was observed when compared to the control liposomes. Although partial inhibition of oxygen photoconsumption observed in the presence of cholesteryl esters may indicate their antioxidant role, it seems that structural effects induced by cholesteryl esters in the lipid membranes are responsible for the observed reduction in oxygen consumption rate in liposomes containing (16:0)(22:6)PC. Indeed, ESR studies employing appropriate spin labels indicated that the ester bond was directed towards the surface of the membrane, while the tetracyclic part of $\mathrm{Ch}$ and the rest of the esterified fatty acid were buried in the hydrophobic interior of the bilayer (Grover et al., 1979). Atomistic simulation studies of the location of cholesteryl oleate in LDL indicated that the oleate chains in most of ChO molecules, especially in the LDL core, existed mainly in extended conformations relative to the ring structures. However, those molecules, located close to the lipoprotein surface, were in a kinked conformation, where the angle between the fatty acid chain and the cholesterol molecule was below $60^{\circ}$ (Heikelä et al., 2006). Esters lacking a hydroxyl group are less polar and therefore localize deeper in the membrane. This causes the molecules of other lipids to slide apart, and the change is even more profound. The rest of the oleic acid has a double bond in the chain. This bond occurs in the cis conformation, so the chain of this ester is bent at an angle of $133^{\circ}$ (Weijers, 2016) and takes up more space. Due to changes in the arrangement of lipid molecules in the membrane bilayer, forced in a way by the cholesteryl ester molecules introduced between them, water molecules can penetrate deeper into the membrane. Considering that water dramatically reduces the singlet oxygen lifetime, when compared to the lipid environment (Baier et al., 2005), presence of water molecules in the membrane could reduce oxidation of the membrane lipids and the corresponding rate of oxygen uptake. It cannot be ruled out that a similar effect was responsible for the reduced oxygen consumption observed in liposomes irradiated in the presence of $\mathrm{RB}$. The presence of Ch slightly accelerated the rate of oxygen uptake in the tested system during exposure to blue light in comparison to the control sample. It should be remembered that cholesterol, also easily oxidized, may act as an additional substrate for ROS generated by partially oxidised (16:0)(22:6)PC exposed to blue light. Interestingly, the lowest $\mathrm{d}\left[\mathrm{O}_{2}\right] / \mathrm{dt}$ in irradiated samples containing partially oxidized (16:0)(22:6) PC was observed in the presence of cholesterol derivatives characterised by the highest interaction rate constant with singlet oxygen.

\section{Singlet oxygen quenching measurements}

Results of the determined singlet oxygen quenching rate by cholesterol, its selected esters, oxidation products and metabolites are presented in Table 3. Saturated lipids interact with singlet oxygen only physically, while unsaturated lipids, including cholesterol and its peroxidisable derivatives/analogues, interact with ${ }^{1} \mathrm{O}_{2}\left({ }^{1} \Delta_{\mathrm{g}}\right)$ both chemically and physically (Bacellar \& Baptista, 2019). A condition for chemical interaction with singlet oxygen is the presence of a double bond which the singlet oxygen molecule can attack. Among the examined cholesterol derivatives, the double bond was present only in $\mathrm{Ch}$ itself, cholesteryl esters and $7 \mathrm{KCh}$. The determined rate constant of the interaction of $\mathrm{Ch}$ with singlet oxygen, $\mathrm{kq}$ is $3.64 \times 10^{4} \mathrm{M}^{-1} \mathrm{~s}^{-1}$. This value does not differ substantially from the literature data (Broniec et al., 2011). Vever-Bizet et al., determined (by the same method) the rate constant for the reaction of cholesterol with ${ }^{1} \mathrm{O}_{2}$ in benzene to be $5.7 \times 10^{4} \mathrm{M}^{-1} \mathrm{~s}^{-1}$ (Vever-Bizet et al., 1989). As expected, the rate constants of $\mathrm{HSCh}$ and $\mathrm{PCh}$ interactions' with singlet oxygen were almost identical to that determined for $\mathrm{Ch}$ alone and were as follows:

Table 3. Rate constants for interactions of ${ }^{1} \mathrm{O}_{2}\left({ }^{1} \Delta_{\mathrm{g}}\right)$ with cholesterol and selected cholesteryl eters, cholesterol oxidation products and cholesterol metabolites determined in chloroform.

TPP has been used as an efficient singlet oxygen generator excited with $645 \mathrm{~nm}$ laser pulse.

Cholesterol

\begin{tabular}{lcc} 
& cholesteryl palmitate & $(3.53 \pm 0.47) \times 10^{4}$ \\
Cholesteryl esters & cholesteryl oleate & $(6.46 \pm 0.42) \times 10^{4}$ \\
& cholesteryl hemisuccinate & $(3.63 \pm 0.42) \times x 10^{4}$ \\
\hline & 7 -ketocholesterol & $(1.31 \pm 0.42) \times \times 10^{4}$ \\
Cholesterol oxidation products & cholesterol epoxide & $(1.16 \pm 0.08) \times 10^{4}$ \\
& cholestanol & $(1.30 \pm 0.03) \times 10^{4}$ \\
Cholesterol metabolities & cholestane & $(1.02 \pm 0.14) \times 10^{4}$ \\
& &
\end{tabular}


$3.63 \times 10^{4} \mathrm{M}^{-1} \mathrm{~s}^{-1}$ and $3.53 \times 10^{4} \mathrm{M}^{-1} \mathrm{~s}^{-1}$, respectively. In the case of the cholesteryl oleate molecule, the constant of the interaction rate with ${ }^{1} \mathrm{O}_{2}$ was almost twice as high and amounted to $6.46 \times 10^{4} \mathrm{M}^{-1} \mathrm{~s}^{-1}$. This is because an additional double bond appeared in the molecule of this cholesteryl ester, located in the esterified oleic acid (18: 1) chain. The determined rate constant of the interaction of $\mathrm{OCh}$ with ${ }^{1} \mathrm{O}_{2}$ also did not differ from the literature $\mathrm{kq}$ values determined for the oleic acid and oleic acid methyl ester, which were: $5.3 \times 10^{4} \mathrm{M}^{-1} \mathrm{~s}^{-1}$ and $7.4 \times 10^{4} \mathrm{M}^{-1} \mathrm{~S}^{-1}$, respectively (Vever-Bizet et al., 1989). In the case of $7 \mathrm{KCh}$, despite the presence of a double bond in the sterol molecule, the $\mathrm{kq}$ is low, amounting to $1.31 \times 10^{4} \mathrm{M}^{-1} \mathrm{~s}^{-1}$. This value corresponds to the constant rate of physical interaction of sterols with singlet oxygen, which were recorded for cholestanol, cholestane and cholesterol epoxide, $1.3 \times 10^{4} \mathrm{M}^{-1} \mathrm{~s}^{-1}, 1.02 \times 10^{4} \mathrm{M}^{-1} \mathrm{~s}^{-1}$ and $1.164 \times 10^{4} \mathrm{M}^{-1} \mathrm{~s}^{-1}$, respectively. It is known, however, that $\alpha, \beta$-unsaturated ketones, especially those in which the carbonyl group is in trans conformation with respect to the double bond, as is the case with $7 \mathrm{KCh}$, have low reactivity towards ${ }^{1} \mathrm{O}_{2}$ (Ensley et al., 1980). Rate constant of singlet oxygen quenching for DMPC, which physically interacts with ${ }^{1} \mathrm{O}_{2}$, was low and amounted to approx. $(1.76 \pm 0.15) \times 10^{4} \mathrm{M}^{-1} \mathrm{~s}^{-1}$, while rates determined for POPC and (16:0)(22:6)PC were higher and were $(5.2 \pm 0.45) \times 10^{4} \mathrm{M}^{-1} \mathrm{~s}^{-1}$ and $(2.08 \pm 0.10) \times 10^{5} \mathrm{M}^{-1} \mathrm{~s}^{-1}$, respectively.

The obtained rate constants of the selected lipids and sterols interaction with singlet oxygen led to a conclusion that the tested compounds were not efficient singlet oxygen quenchers. The obtained values $\left(\sim 10^{4} \quad \mathrm{M}^{-1} \mathrm{~s}^{-1}\right)$ were much lower than those reported for typical for biological antioxidants $\left(10^{8}-10^{10} \mathrm{M}^{-1} \mathrm{~s}^{-1}\right)$ (Cantrell et al., 2003; Gruszka et al., 2008).

\section{Acknowledgement}

We are grateful to Professor H.J. Halpern for providing us with the mHCTPO nitroxide probe.

\section{REFERENCES}

Anderson A, Campo A, Fulton E, Corwin A, Jerome WG, 3rd, O'Connor MS (2020) 7-Ketocholesterol in disease and aging. Redox Biol 29: 101380-101380. https://doi.org/10.1016/j.redox.2019.101380

Bacellar IOL, Baptista MS (2019) Mechanisms of photosensitized lipid oxidation and membrane permeabilization. ACS Omega 4: 2163621646. https://doi.org/10.1021/acsomega.9b03244

Baier J, Maier M, Engl R, Landthaler M, Bäumler W (2005) Timeresolved investigations of singlet oxygen luminescence in water, in phosphatidylcholine, and in aqueous suspensions of phosphatidylcholine or HT29 cells. J Phys Chem B 109: 3041-3046. https://doi. org/10.1021/jp0455531

Bhattacharyya AK, Lin DS, Connor WE (2007) Cholestanol metabolism in patients with cerebrotendinous xanthomatosis: absorption, turnover, and tissue deposition. J Lipid Res 48: 185-192. https:// doi.org/10.1194/jlr.M600113-JLR200

Bonnett R, McGarvey DJ, Harriman A, Land EJ, Truscott TG, Winfield UJ (1988) Photophysical properties of meso-tetraphenylporphyrin and some meso-tetra(hydroxyphenyl)porphyrins. Photochem Photobiol 48: 271-276

Broniec A, Klosinski R, Pawlak A, Wrona-Krol M, Thompson D, Sarna T (2011) Interactions of plasmalogens and their diacyl analogs with singlet oxygen in selected model systems. Free Radic Biol Med 50: 892-898. https://doi.org/10.1016/j.freeradbiomed.2011.01.002

Brown MS, Goldstein JL (1983) Lipoprotein metabolism in the macrophage: implications for cholesterol deposition in atherosclerosis. Annu Rev Biochem 52: 223-261. https://doi.org/10.1146/annurev. bi.52.070183.001255

Cantrell A, McGarvey DJ, Truscott TG, Rancan F, Böhm F (2003) Singlet oxygen quenching by dietary carotenoids in a model membrane environment. Arch Biochem Biophys 412: 47-54. https://doi. org/10.1016/s0003-9861(03)00014-6
Crandon LE, Boenisch KM, Harper BJ, Harper SL (2020) Adaptive methodology to determine hydrophobicity of nanomaterials in situ. PLOS ONE 15: e0233844. https://doi.org/10.1371/journal. pone. 0233844

DeRosa MC, Crutchley RJ (2002) Photosensitized singlet oxygen and its applications. Coord Chem Rev 233-234: 351-371. https://doi. org/10.1016/S0010-8545(02)00034-6

Dotti MT, Rufa A, Federico A (2001) Cerebrotendinous xanthomatosis: Heterogeneity of clinical phenotype with evidence of previously undescribed ophthalmological findings. I Inh Metabol Dis 24: 696706. https://doi.org/10.1023/A:1012981019336

Enomoto M, Nakagami K, Ohkuma S, Takano T (1987) Transformation of macrophages into foam cells in vitro induced by cholesteryl oleate liquid crystals. J Biochem 101: 933-938. https://doi. org/10.1093/oxfordjournals.jbchem.a121962

Ensley HE, Carr RVC, Martin RS, Pierce TE (1980) Reaction of singlet oxygen with alpha,beta-unsaturated ketones and lactones. $J \mathrm{Am}$ Chem Soc 102: 2836-2838. https://doi.org/10.1021/ja00528a053

Geiger PG, Korytowski W, Lin F, Girotti AW (1997) Lipid peroxidation in photodynamically stressed mammalian cells: use of cholesterol hydroperoxides as mechanistic reporters. Free Radic Biol Med 23: 57-68. https://doi.org/10.1016/s0891-5849(96)00587-4

Grover AK, Forrest BJ, Buchinski RK, Cushley RJ (1979) ESR studies on the orientation of cholesteryl ester in phosphatidylcholine multilayers. Biochim Biophys Acta - Biomembranes 550: 212-221. https://doi. org/10.1016/0005-2736(79)90208-6

Gruszka J, Pawlak A, Kruk J (2008) Tocochromanols, plastoquinol, and other biological prenyllipids as singlet oxygen quenchers-determination of singlet oxygen quenching rate constants and oxidation products. Free Radic Biol Med 45: 920-928. https://doi.org/10.1016/j. freeradbiomed.2008.06.025

Heikelä M, Vattulainen I, Hyvönen MT (2006) Atomistic simulation studies of cholesteryl oleates: model for the core of lipoprotein particles. Biophys J 90: 2247-2257. https://doi.org/10.1529/biophysj.105.069849

Jeffrey BG, Weisinger HS, Neuringer M, Mitchell DC (2001) The role of docosahexaenoic acid in retinal function. Lipids 36: 859-871. https://doi.org/10.1007/s11745-001-0796-3

Koivuniemi A, Heikelä M, Kovanen PT, Vattulainen I, Hyvönen MT (2009) Atomistic simulations of phosphatidylcholines and cholesteryl esters in high-density lipoprotein-sized lipid droplet and trilayer: clues to cholesteryl ester transport and storage. Biophys J 96: 4099_ 4108. https://doi.org/10.1016/j.bpj.2009.01.058

Koscielniak A, Serafin M, Duda M, Oles T, Zadlo A, Broniec A, Berdeaux O, Gregoire S, Bretillon L, Sarna T, Pawlak A (2017) Oxidation-induced increase in photoreactivity of bovine retinal lipid extract. Cell Biochem Biophys 75: 443-454. https://doi.org/10.1007/ s12013-017-0832-3

Kulig W, Cwiklik L, Jurkiewicz P, Rog T, Vattulainen I (2016) Cholesterol oxidation products and their biological importance. Chem Phys Lipids 199: 144-160. https://doi.org/10.1016/j.chemphyslip.2016.03.001

Lambert CR, Kochevar IE (1997) Electron transfer quenching of the rose bengal triplet state. Photochem Photobiol 66: 15-25. https://doi. org/10.1111/j.1751-1097.1997.tb03133.x

Liu Y, Zhang D, Wu Y, Ji B (2014) Docosahexaenoic acid aggravates photooxidative damage in retinal pigment epithelial cells via lipid peroxidation. J Photochem Photobiol B: Biol 140: 85-93. https://doi. org/10.1016/j.jphotobiol.2014.07.016

MacDonald RC, MacDonald RI, Menco BP, Takeshita K, Subbarao NK, Hu LR (1991) Small-volume extrusion apparatus for preparation of large, unilamellar vesicles. Biochim Biophys Acta 1061: 297303. https://doi.org/10.1016/0005-2736(91)90295-j

Nagano Y, Arai H, Kita T (1991) High density lipoprotein loses its effect to stimulate efflux of cholesterol from foam cells after oxidative modification. PNAS 88: 6457-6461. https://doi.org/10.1073/ pnas.88.15.6457

Nayar R, Hope MJ, Cullins PR (1989) Generation of large unilamellar vesicles from long-chain saturated phosphatidylcholines by extrusion technique. Biochim Biophys Acta - Biomembranes 986: 200-206. https:// doi.org/10.1016/0005-2736(89)90468-9

Neckers DC (1989) Rose Bengal. J Photochem Photobiol A: Chem 47: 1-29. https://doi.org/10.1016/1010-6030(89)85002-6

Pawlak AM, Olchawa M, Koscielniak A, Zadlo A, Broniec A, Oles T, Sarna TJ (2019) Oxidized lipids decrease phagocytic activity of ARPE-19 cells in vitro. Eur J Lipid Sci Technol 121: 1800476. https:// doi.org/10.1002/ejlt.201800476

Rao X, Zhong J, Maiseyeu A, Gopalakrishnan B, Villamena FA, Chen LC, Harkema JR, Sun Q, Rajagopalan S (2014) CD36-dependent 7-ketocholesterol accumulation in macrophages mediates progression of atherosclerosis in response to chronic air pollution exposure. Circ Res 115: 770-780. https://doi.org/10.1161/CIRCRESAHA.115.304666

Redmond RW, Gamlin JN (1999) A compilation of singlet oxygen yields from biologically relevant molecules. Photochem Photobiol 70: $391-475$ 
Rodgers MAJ, Snowden PT (1982) Lifetime of oxygen (O2(1. DELTA.g)) in liquid water as determined by time-resolved infrared luminescence measurements. J Am Chem Soc 104: 5541-5543. https://doi.org/10.1021/ja00384a070

Rodriguez IR, Clark ME, Lee JW, Curcio CA (2014) 7-Ketocholesterol accumulates in ocular tissues as a consequence of aging and is present in high levels in drusen. Exp Eye Res 128: 151-155. https:// doi.org/10.1016/j.exer.2014.09.009

Rodriguez IR, Fliesler SJ (2009) Photodamage generates 7-keto- and 7-hydroxycholesterol in the rat retina via a free radical-mediated mechanism. Photochem Photobiol 85: 1116-1125. https://doi. org/10.1111/j.1751-1097.2009.00568.x

Rozanowska M, Jarvis-Evans J, Korytowski W, Boulton ME, Burke JM, Sarna T (1995) Blue light-induced reactivity of retinal age pigment. In vitro generation of oxygen-reactive species. J Biol Chem 270 : 18825-18830

Różanowska MB, Pawlak A, Różanowski B (2021) Products of docosahexaenoate oxidation as contributors to photosensitising properties of retinal lipofuscin. Int J Mol Sci 22. https://doi.org/10.3390/ ijms 22073525

Różanowska MO, Ciszewska J, Korytowski W, Sarna T (1995) Rosebengal-photosensitized formation of hydrogen peroxide and hydroxyl radicals. J Photochem Photobiol B: Biol 29: 71-77. https://doi. org/10.1016/1011-1344(95)90259-7

Seyama Y (2003) Cholestanol metabolism, molecular pathology, and nutritional implications. J Med Food 6: 217-224. https://doi. org/10.1089/10966200360716634

Stockett MH, Kjær C, Daly S, Bieske EJ, Verlet JRR, Nielsen SB, Bull JN (2020) Photophysics of isolated rose bengal anions. J Physic Chem A 124: 8429-8438. https://doi.org/10.1021/acs.jpca.0c07123

Subczynski WK, Pasenkiewicz-Gierula M, Widomska J, Mainali L, Raguz M (2017) High cholesterol/low cholesterol: effects in biological membranes: a review. Cell Biochem Biophys 75: 369-385. https:// doi.org/10.1007/s12013-017-0792-7
Subczynski WK, Raguz M, Widomska J, Mainali L, Konovalov A (2012) Functions of cholesterol and the cholesterol bilayer domain specific to the fiber-cell plasma membrane of the eye lens. J Membr Biol 245: 51-68. https://doi.org/10.1007/s00232-011-9412-4

Subczynski WK, Wisniewska A, Hyde JS, Kusumi A (2007) Three-dimensional dynamic structure of the liquid-ordered domain in lipid membranes as examined by pulse-EPR oxygen probing. Biophys $J$ 92: 1573-1584. https://doi.org/10.1529/biophysj.106.097568

Vever-Bizet C, Dellinger M, Brault D, Rougee M, Bensasson RV (1989) Singlet molecular oxygen quenching by saturated and unsaturated fatty-acids and by cholesterol. Photochem Photobiol 50: 321-325. https://doi.org/10.1111/j.1751-1097.1989.tb04165.x

Vila A, Korytowski W, Girotti AW (2001) Spontaneous intermembrane transfer of various cholesterol-derived hydroperoxide species: kinetic studies with model membranes and cells. Biochemistry 40: 1471514726. https://doi.org/10.1021/bi011408r

Weijers RNM (2016) Membrane flexibility, free fatty acids, and the onset of vascular and neurological lesions in type 2 diabetes. I Diabetes Metab Disord 15: 13-13. https://doi.org/10.1186/s40200-016-0235-9

Wnętrzak A, Makyła-Juzak K, Filiczkowska A, Kulig W, DynarowiczŁątka P (2017) Oxysterols versus cholesterol in model neuronal membrane. i. the case of 7-ketocholesterol. the langmuir monolaver study. J Membr Biol 250: 553-564. https://doi.org/10.1007/s00232017-9984-8

Yang ST, Kreutzberger AJB, Lee J, Kiessling V, Tamm LK (2016) The role of cholesterol in membrane fusion. Chem Phys Lipids 199: 136143. https://doi.org/10.1016/j.chemphyslip.2016.05.003

Zadlo A, Burke JM, Sarna T (2009) Effect of untreated and photobleached bovine RPE melanosomes on the photoinduced peroxidation of lipids. Photochem Photobiol Sci 8: 830-837. https://doi. org/10.1039/b901820d 